\title{
MEDICO - SOCIAL SUBSTANTIATION OF THE CONCEPT OF PERSONALIZED MEDICINE IN THE PREVENTION OF ARTERIAL HYPERTENSION AMONG THE ADULT POPULATION AT THE REGIONAL LEVEL
}

D0I: 10.36740/WLek202103216

\author{
Zinaida V. Lashkul', Dmytro A. Lashkul ${ }^{2}$ \\ 'STATE INSTITUTION “ZAPORIZHZHIA MEDICAL ACADEMY OF POSTGRADUATE EDUCATION OF THE MINISTRY OF HEALTH OF UKRAINE,"ZAPORIZHZHIA, UKRAINE \\ 2ZAPORIZHZHIA STATE MEDICAL UNIVERSITY, ZAPORIZHZHIA, UKRAINE
}

\begin{abstract}
The aim: Is to scientifically substantiate the concept of personalized medicine in the prevention of arterial hypertension (AH) among the adult population at the regional level. Materials and methods: The study, after obtaining informed consent, involved 2000 patients, residents of Zaporizhzhia Region, including 1000 patients (average age $59 \pm 0.5$ years, the ratio of men to women 1:1.22 people) with and 1000 patients (average age of whom is $62 \pm 0.7$ years, the ratio of men to women is 1:1.1) without AH. The data of the sociological survey were processed, the methods of mathematical modeling, statistical, system analysis were applied. Odds ratio (OR) is determined by generally accepted methods with the calculation of $95 \%$ of confidence interval (Cl).

Results: The application of the concept of personalized medicine in the prevention of AH among the adult population has been scientifically substantiated by studying the associations between medical and social risk factors and the development of AH and determining on their basis 3 modules - "risk behavior", "adverse health factors" and "social determinants of the development of $\mathrm{AH}^{\prime \prime}$, based on stepwise multiple logistic regression analysis.

Conclusions: It has been proved that the use of the final prognostic model of the development of arterial hypertension with the inclusion of the most significant risk factors has high operational characteristics: sensitivity $-78.6 \%$, specificity $-96.6 \%$, positive predicative value $-95.85 \%$, negative predicative value $-81.86 \%$, the area under the ROC curve is 0.9623 . The application of the concept of personalized medicine in the prevention of this disease among the adult population was substantiated by studying the associations between medical and social risk factors and the development of arterial hypertension.
\end{abstract}

KEY WORDS: risk factors, arterial hypertension, personalized medicine

Wiad Lek. 2021;74(3 p.II):652-657

\section{INTRODUCTION}

The profile of risk factors (RFs) among different segments of the population may differ, which justifies the need to develop a system for the prevention of arterial hypertension $(\mathrm{AH})$ for each individual with the determination of individual RFs. In this aspect it is interesting the theory of ideal cardiovascular health [1-6], which shifts the priorities for determining the RFs of the development of circulatory system diseases (CSD) to health factors of the cardiovascular system (CVS), in particular, the concept "risky" behavior that increases the likelihood of developing CVD and stroke, or conditions that lead to their development, such as hypertension, dyslipidemia and diabetes, are changed to "healthy" behavior.

Currently, the definition of "ideal cardiovascular health" is well known and is characterized by the simultaneous presence of 4 criteria for "healthy" behavior: abstaining from smoking during the last year, ideal body mass index $\left[<25 \mathrm{~kg} / \mathrm{m}^{2}\right]$, recommended physical activity, consumption an appropriate set of products that prevent the development of CVD and 3 favorable health factors: total cholesterol $<200 \mathrm{mg} /$ or $(4.5 \mathrm{mmol} / \mathrm{l})$, blood pressure $<120 /<80 \mathrm{~mm}$ $\mathrm{Hg}$, the absence of diabetes mellitus and clinical manifestations of CSD, including coronary heart disease (CHD), stroke, heart failure, etc. $[7,8,9]$.

\section{THE AIM}

The aim of the work is to scientifically substantiate the concept of personalized medicine in the prevention of arterial hypertension among the adult population at the regional level.

\section{MATERIALS AND METHODS}

Methods of mathematical modeling, statistical, system analysis were used. In the course of the study, the need to implement the concept of personalized medicine into the system of primary and secondary prevention of arterial hypertension was substantiated by studying and identifying 
Table I. Associations between risk factors and the development of hypertension among the adult population, $\mathrm{n}(\%)$

\begin{tabular}{|c|c|c|c|c|}
\hline Risk factors & $\begin{array}{c}\text { Individuals with } \\
\text { AH } n=1000\end{array}$ & $\begin{array}{c}\text { Individuals without } \\
\mathrm{AH} \mathrm{n}=1000\end{array}$ & OR $(95 \% \mathrm{Cl})$ & $\mathbf{p}$ \\
\hline Social status - employee & $878(87.8)$ & $740(74.0)$ & $2.53(1.99-3.205)$ & $<0.001$ \\
\hline Low level of education & $423(42.3)$ & $234(23.4)$ & $2.39(1.97-2.91)$ & $<0.0001$ \\
\hline Salary < 1900 UAH & $664(66.4)$ & $342(34.2)$ & $3.80(3.15-4.57)$ & $<0.0001$ \\
\hline Poor living standards & $670(67.0)$ & $453(45.3)$ & $2.45(2.04-2.94)$ & $<0.0001$ \\
\hline Stress-related profession & $320(32.0)$ & $320(32)$ & $1.00(0.83-1.21)$ & 1.00 \\
\hline Unemployed & 122 & 68 & $1.9(1.38-2.64)$ & $<0.0001$ \\
\hline Loneliness & $264(26.4)$ & $265(26.5)$ & $0.99(0.82-1.21)$ & 0.960 \\
\hline Low average income & $670(67.0)$ & $132(13.20)$ & $13.35(10.65-16.73)$ & $<0.0001$ \\
\hline Lack of healthcare facility at the place of residence & $116(11.60)$ & $320(32.0)$ & $0.29(0.22-0.35)$ & $<0.0001$ \\
\hline Salt intake $>5 \mathrm{~g}$ per day & $524(52.4)$ & $64(6.4)$ & $16.09(12.14-21.34)$ & $<0.0001$ \\
\hline Excessive consumption of fatty meats, fish daily & $423(42.3)$ & $342(34.2)$ & $1.41(1.18-1.69)$ & $<0.0001$ \\
\hline Insufficient consumption of vegetables and fruits & $854(86.4)$ & $245(24.5)$ & $18.02(14.36-22.62)$ & $<0.0001$ \\
\hline Level of glucose in blood $>6.0 \mathrm{mmol} / \mathrm{L}$ & $158(15.8)$ & $66(6.6)$ & $2.65(1.96-3.59)$ & $<0.0001$ \\
\hline Cholesterol level $>4.5 \mathrm{mmol} / \mathrm{L}$ & $363(36.3)$ & $152(15.2)$ & $3.18(2.56-3.94)$ & $<0.0001$ \\
\hline
\end{tabular}

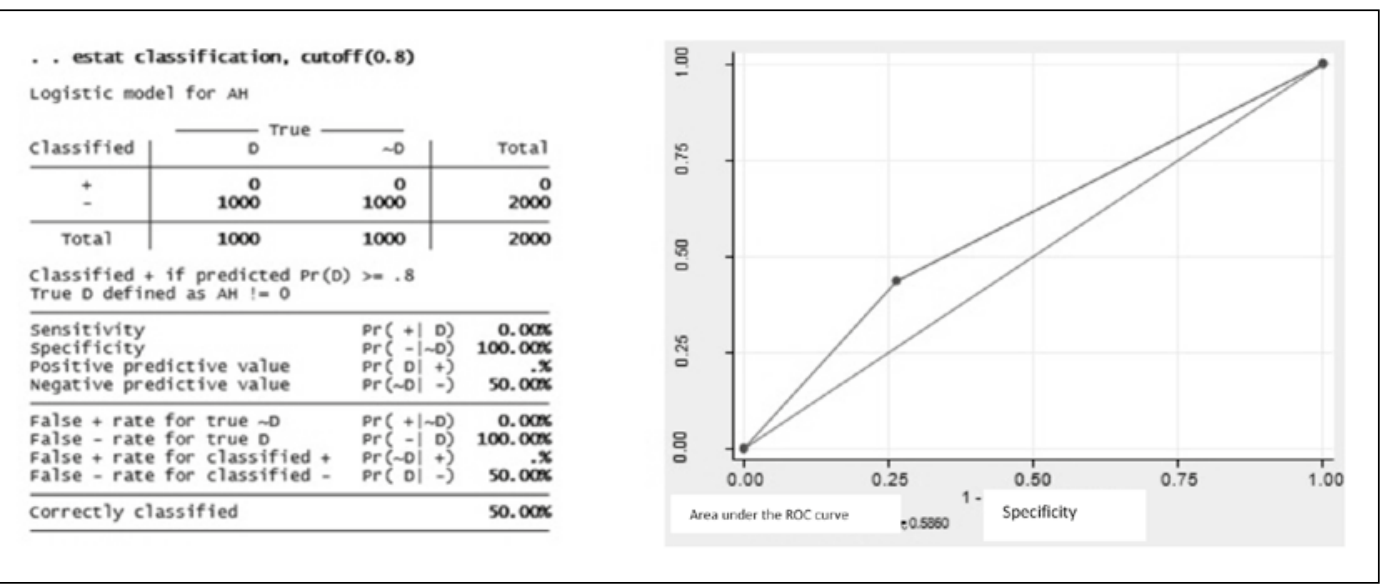

Fig. 1. Operational characteristics of the predictive model, which includes 1 risk factor - lack of physical activity and the ROC-curve of the predictive model, containing 1 risk factor lack of physical activity

Fig. 2. Operational characteristics of the predictive model, which includes 2 risk factors - lack of physical activity and overweight and the ROC curve of the predictive model, which contains 2 risk factors - lack of physical activity and overweight the most significant factors that are reliably associated with the development of the disease and developing clinical prognostic models of arterial hypertension with the calculation of operational characteristics and the construction of ROC curves.
For this, a prospective cohort study was carried out, which included 1000 patients with arterial hypertension treated in the 9th City Hospital of the Zaporizhzhia (average age $59 \pm 0.5$ years, the ratio of men to women 1:1.22 people) and 1000 people without arterial hypertension (average age of whom 

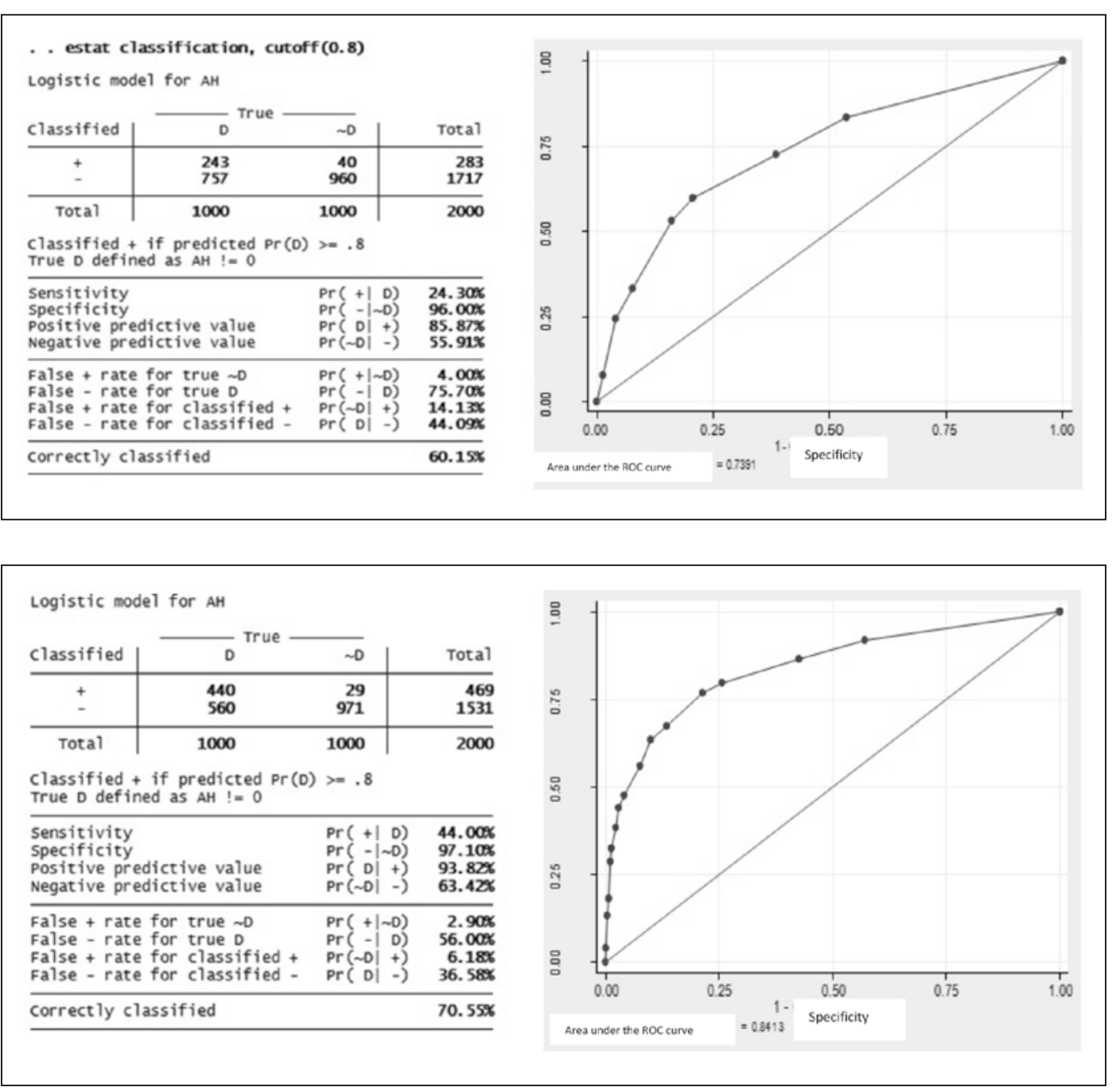

Fig.3. The operational characteristics of the predictive model, which includes 3 risk factors - lack of physical activity, overweight and smoking, and the ROC curve of the predictive model, contains 3 risk factors - lack of physical activity, overweight and smoking

Fig. 4. Operational characteristics of the predictive model, which includes 4 risk factors - lack of physical activity, overweight, smoking and excessive salt intake and the ROC curve of the predictive model containing 4 risk factors - lack of physical activity, overweight, smoking and excessive salt intake is $62 \pm 0.7$ years, the ratio of men to women is 1:1.1). In total, 20 prognostic variables were considered, probably associated with the development of arterial hypertension.The identified risk factors were significantly associated with the development of arterial hypertension in simple logistic regression analysis, were subjected to multiple logistic regression analysis to identify reliable risk factors, which later became the basis for the development of an algorithm for identifying high-risk patients. The generally accepted methods were used to determine the odds ratio (OR) based on the $95 \%$ confidence interval (CI).

\section{RESULTS}

The initial stage of the study was to study the association between RFs and the development of $\mathrm{AH}$ in the adult population. It was found that the development of $\mathrm{AH}$ among the population of the Zaporizhzhia region is most affected by a burdened family history, therefore, such a criterion as the absence of CSD in close relatives was necessarily added to the favorable health factors.

The definition "Ideal cardiovascular health" as a separate module includes the social determinants of health - a satisfactory standard of living, which implies an adequate average standard of living and a decent salary.
According to the experts of the World Health Organization, the morbidity and mortality rates from CSD are influenced by: low standard of living of the population; associated unemployment, stress, alcoholism; lack of motivation among the population to be healthy; lack of motivation among medical workers to work efficiently, low level of material and technical base of health care, insufficient funding of the industry [3].

In our study, among the surveyed respondents without $\mathrm{AH}$ in the surveyed cohort (1000 persons without $\mathrm{AH}$ ), only 56 people had ideal cardiovascular health (Table 1). According to the data, the prognostic model of the development of AH includes the criteria of all 3 modules: "crisis behavior", "adverse health factors" and "social determinants of the development of AH".

To determine the risk of the development of $\mathrm{AH}$ in an individual with the cumulative effect of several factors, a stepwise multiple logistic regression method based on risk calculations is used, combining and integrating premorbid conditions, accessible and minimally intrusive demographic and medical criteria that are easily understood by individuals and are available in medical institutions. The first predictive model, which includes only one RF - lack of physical activity, has low operational characteristics (Fig. 1). 
Table II. Clinical prognostic models for the development of AH in the adult population

\begin{tabular}{|c|c|c|c|c|c|c|c|}
\hline Prognostic changes & $\boldsymbol{\beta}$ & $\mathbf{m}$ & OR & $95 \% \mathrm{Cl}$ & $\mathbf{p}$ & $\begin{array}{l}\text { Area under the } \\
\text { ROC curve }\end{array}$ & $\begin{array}{c}\% \text { of area } \\
\text { increase }\end{array}$ \\
\hline \multicolumn{8}{|c|}{ "Risky behavior" } \\
\hline Lack of physical activity & 0.768 & 0.095 & 2.15 & $1.78-2.60$ & $<0.001$ & 0.5860 & \\
\hline Overweight & 1.76 & 0.108 & 5.83 & $4.72-7.21$ & $<0.001$ & 0.723 & 23.4 \\
\hline Smoking & 0.51 & 0.109 & 1.68 & $1.35-2.08$ & $<0.001$ & 0.739 & 2.2 \\
\hline Salt abuse & 2.73 & 0.152 & 15.42 & $11.44-20.78$ & $<0.001$ & 0.8413 & 13.8 \\
\hline \multicolumn{8}{|c|}{ «Unfavorable health» } \\
\hline Family history of hypertension & 3.91 & 0.18 & 50.01 & $35.10-71.24$ & $<0.001$ & 0.9439 & 12.2 \\
\hline High cholesterol level & 0.76 & 0.18 & 2.14 & $1.49-3.08$ & $<0.001$ & 0.946 & 0.2 \\
\hline High glucose level & 0.79 & 0.25 & 2.20 & 1.34-3.59 & 0.002 & 0.9478 & 0.2 \\
\hline \multicolumn{8}{|c|}{ «Social determinants of the development of $A H »$} \\
\hline Low income & 2.63 & 0.187 & 13.87 & $9.61-20.04$ & $<0.001$ & 0.9674 & 2.1 \\
\hline
\end{tabular}

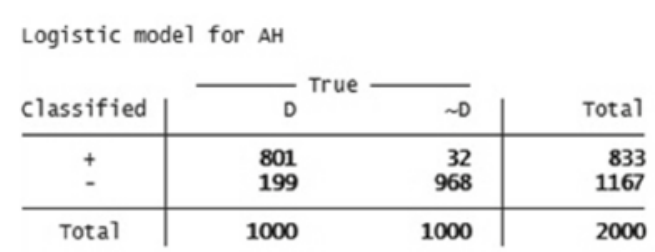

Classified + if predicted $\operatorname{Pr}(D)>=.8$

True $\mathrm{D}$ defined as $\mathrm{AH} !=0$

\begin{tabular}{llr}
\hline Sensitivity & $\operatorname{Pr}(+\mid$ D) & $\mathbf{8 0 . 1 0 \%}$ \\
Specificity & $\operatorname{Pr}(-\mid \sim D)$ & $\mathbf{9 6 . 8 0 \%}$ \\
Positive predictive value & $\operatorname{Pr}(\mathrm{D} \mid+)$ & $\mathbf{9 6 . 1 6 \%}$ \\
Negative predictive value & $\operatorname{Pr}(\sim D \mid-)$ & $\mathbf{8 2 . 9 5 \%}$ \\
\hline False + rate for true $\sim 0$ & $\operatorname{Pr}(+\mid \sim D)$ & $\mathbf{3 . 2 0 \%}$ \\
False - rate for true D & $\operatorname{Pr}(-\mid D)$ & $\mathbf{1 9 . 9 0 \%}$ \\
False + rate for classified + & $\operatorname{Pr}(\sim 0 \mid+)$ & $\mathbf{3 . 8 4 \%}$ \\
False - rate for classified - & $\operatorname{Pr}(\mathrm{D} \mid-)$ & $\mathbf{1 7 . 0 5 \%}$ \\
\hline correctly classified & & $\mathbf{8 8 . 4 5 \%}$
\end{tabular}
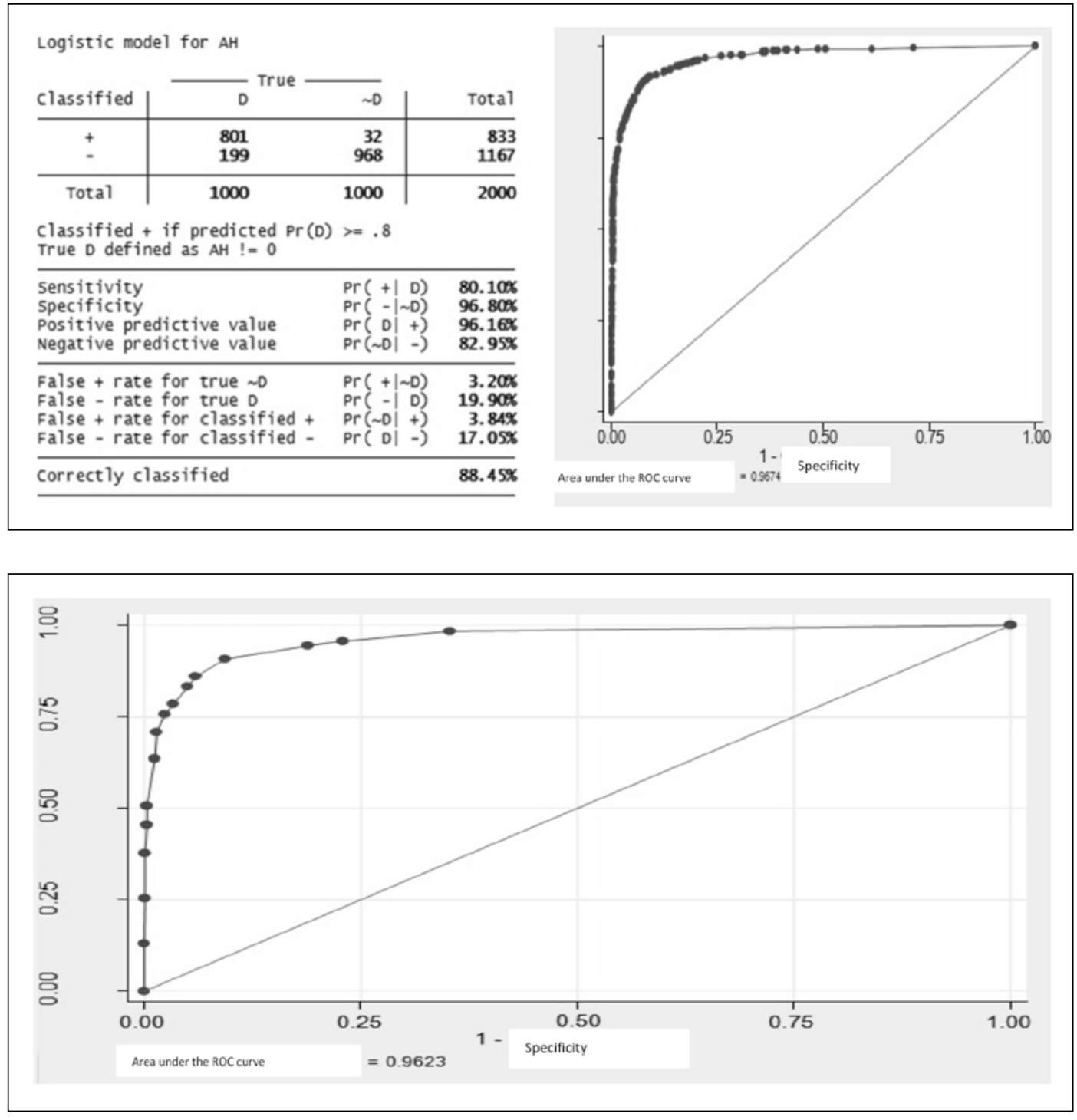

Fig. 5. Operational characteristics of the predictive model, which includes 8 risk factors and the ROC curve of the predictive model, which contains 8 risk factors

Fig. 6. Predictive model of the development of $\mathrm{AH}$ in the adult population
The addition of the following RF - overweight - has a significant effect on the development of $\mathrm{AH}$ such a factor as lack of physical activity, and somewhat in- creases the operational characteristics of the model, in particular, the area under the ROC-curve increases by $23.4 \%$ (Fig. 2). 
The addition of a third RF from the module "risky behavior" to the predictive model - smoking - increases the model's characteristics by only $2.2 \%$, but RF - excessive salt intake - by $13.8 \%$, leaving all previous RFs significant (Fig. 3).

Thus, in multiple logistic regression analysis, all of the above RFs of the "risky behavior" module are reliably associated with the development of $\mathrm{AH}$. But overweight and excessive salt intake have the greatest influence on the development of $\mathrm{AH}$. The inclusion of all 4 risk factors from the "risky behavior" module significantly increases the area under the ROC curve, indicating high predictive characteristics of such a model (Fig. 4).

The next RFs added to the predictive model are factors from the module "adverse health factors". According to the data of the table 2, the addition of such RF as family history of hypertension significantly increases the characteristics of the model, and the area under the ROC curve increases immediately by $12.2 \%$ - from 0.8413 to 0.9439 .

However, adding "high glucose level" or "high cholesterol level" to the RFs model increases the predictive value of the model by only $0.2 \%$. Thus, it is the RF of the "risky behavior" module and the burdened heredity that are most often associated with the development of $\mathrm{AH}$, and the appointment of additional research methods does not increase the predictive power of the model. From the module "social determinants of the development of $\mathrm{AH}$ ", only one RF is included - low income, of all the factors of the indicated module, it has the highest OR of the development of $\mathrm{AH}(\mathrm{OR}-13.35)$. At the same time, the operational characteristics of the predictive model, which includes 8 risk factors, increase by $2.1 \%$ (Fig. 5).

Thus, the additional inclusion of other risk factors in the model only complicates the calculations but does not improve forecasting. Therefore, our final model includes the RFs most associated with the development of $\mathrm{AH}$ : overweight, high dietary salt intake, family history of hypertension and low income, that is, factors that can be determined by direct conversation with the patient, or factors that each individual can determine in himself directly. The predictive model, which includes only the above factors, has high operational characteristics: sensitivity $-78.6 \%$, specificity - $96.6 \%$, positive predictive value $-95.85 \%$, negative predictive value $-81.86 \%$, area under the ROC curve -0.9623 , which is only 0.0051 less than the predictive model, which includes 8 RFs (Fig. 6).

Thus, when planning a prevention system, it is of paramount importance to determine whether an individual patient has RF from the "crisis behavior" module, and the developed measures should be aimed at modifying the existing RFs.

\section{DISCUSSION}

Our study showed that prevention of AH should be based on finding out whether patients have RF of 3 key modules:

- "healthy behavior" - abstinence from smoking during the last year, ideal body mass index $\left[<25 \mathrm{~kg} / \mathrm{m}^{2}\right]$, recommended physical activity, consumption of an appropriate set of foods that prevent the development of CVD;

- "favorable health factors" - total cholesterol $<200 \mathrm{mg}$ for $(4.5 \mathrm{mmol} / \mathrm{l})$, blood pressure $<120 /<80 \mathrm{~mm} \mathrm{Hg}$, absence of diabetes mellitus and clinical CSD (including CHD, stroke, heart failure, etc.);

- "social determinants of health" - low level of education, low average income, incl. wages $<1900 \mathrm{UAH}$, poor living standarts, associated unemployment, stress, alcoholism, lack of motivation among the population to be healthy, lack of health care facilities at the place of residence.

Therefore, the system of prevention of $\mathrm{AH}$ should be aimed not only at each individual, who has his own individual differences in genes, in the way of life, but also at the population as a whole, which forms his environment. In this aspect, it is the concept of personalized medicine (Precision medicine) that is the tool that will allow influencing each individual in the population $[2,9]$. In early 2015 , the concept of personalized medicine was defined as one of the strategic directions of fundamental research by the US National Institutes of Health, the goal of which is to ensure the health of an individual and increase his life expectancy through the use of preventive or therapeutic measures taking into account individual differences in the genes, environment and lifestyle of the patient. The theory of "ideal cardiovascular health" (American Heart Association Strategic Planning Task Force, 2015), which in recent years has been widely spread in the developed countries of the world, closely intersects with this concept. This theory is based on basic concepts related to the field of health improvement and disease prevention:

1. the importance of primary (primordial) prevention;

2. the presence of evidences that CSD and RFs of their occurrence often develop at an early age;

3. the need for an appropriate balance between approaches to health improvement and disease prevention at the population and individual levels in high-risk individuals, ie focusing on RFs prevention at all levels is paramount, and avoiding adverse RFs levels may be the most effective means of preventing development of the disease throughout life.

Family doctors should make a significant contribution to personalized medicine. The family doctor should take part in the formation of a specific family education program, focused on the tendency of family members to follow the rules of a healthy lifestyle and the prevention of alimentary dependent diseases. The patient should be offered specific health-improving measures, including more often non-pharmacological, stimulating natural mechanisms of repair: the regime of the day and rest, physical exercises, proper nutrition. The patient can receive consultations and assistance in their implementation on the basis of multimodular participation from various public health services, including specialists in food technology, physical culture, tourism, ecology, agrochemistry, etc. 


\section{CONCLUSIONS}

It is proved that the application of the final prognostic model of the development of $\mathrm{AH}$ with the inclusion of the most significant risk factors has high operational characteristics: sensitivity $-78.6 \%$, specificity $-96.6 \%$, positive predicative value $-95.85 \%$, negative predictive value $-81.86 \%$, the area under the ROC curve -0.9623 . The application of the concept of personalized medicine in the prevention of arterial hypertension among the adult population by studying the associations between medical and social risk factors and the development of arterial hypertension is substantiated.

\section{REFERENCES}

1. Williams B., Mancia G., Spiering W. et al. 2018 ESC/ESH Guidelines for the management of arterial hypertension [published correction appears in Eur Heart J. Eur Heart J. 2018; 39(33): 3021-3104. doi:10.1093/ eurheartj/ehy339.

2. NCD Risk Factor Collaboration. Worldwide trends in blood pressure from 1975 to 2015: a pooled analysis of 1479 population-based measurement studies with 19.1 million participants. Lancet. 2017; 389: 37-55.

3. Piepoli M.F., Hoes A.W., Agewall S. et al. ESC Scientific Document Group. 2016 European Guidelines on cardiovascular disease prevention in clinical practice: The Sixth Joint Task Force of the European Society of Cardiology and Other Societies on Cardiovascular Disease Prevention in Clinical Practice (constituted by representatives of 10 societies and by invited experts) Developed with the special contribution of the European Association for Cardiovascular Prevention \& Rehabilitation (EACPR). Eur. Heart J. 2016; 37: 2315-2381.

4. Tiffe T., Wagner M., Rucker V. et al. Control of cardiovascular risk factors and its determinants in the general population - findings from the STAAB cohort study. BMC Cardiovasc. Disord. 2017; 17: 276.

5. Gupta P., Patel P., Strauch B. et al. Biochemical screening for nonadherence is associated with blood pressure reduction and improvement in adherence. Hypertension. 2017; 70: 1042-1048.

6. Dedov I.I. Personalized Medicine. - Annals of the Russian Academy of Medical Sciences. 2019; 74(1):61-70.

7. Report of the USA President's Council of Advisors on Science and Technology. Priorities for Personalized Medicine. 2008 . http:// oncotherapy.us/pdf/ PM.Priorities.pdf.
8. Terkola R., Antoñanzas F., Postma M. Economic evaluation of personalized medicine: a call for real-world data. Eur J Health Econ. 2017; 18(9):1065-1067. doi: 10.1007/s10198-017-0890-x.

9. Kolpachkova E.V., Sokolova A.A., Napalkov D.A. Personalized medicine in cardiology: state, problems and prospects. Meditsinskiy sovet [Medical Council]. 2017; 12: 162-168. (In Russian). https://doi. org/10.21518/2079-701X-2017-12-162-168

The study was performed in the framework of complex research work "Social and hygienic study of patients with hypertension and scientific substantiation of measures to reduce it" (registration No. 02.16.01.09, periods of fulfillment 2014-2019) State Institution "Zaporizhzhia Medical Academy of Postgraduate Education of the Ministry of Health of Ukraine".

\section{ORCID and contributionship:}

Zinaida V. Lashkul: 0000-0002-4693-6803 ${ }^{A, B, C, D}$

Dmytro A. Lashkul: 0000-0001-7802-3550 ${ }^{E, F}$

\section{Conflicts of interest:}

Authors declare no conflict of interest

\section{CORRESPONDING AUTHOR Zinaida V. Lashkul \\ Zaporizhzhia Medical Academy of Postgraduate Education of the Ministry of Health of Ukraine 20 Vintera boul., 69096 Zaporizhzhia, Ukraine tel:+380676026931 \\ e-mail:zlashku|@gmail.com}

Received: 17.11 .2020

Accepted: 08.03 .2021

A - Work concept and design, B - Data collection and analysis, C - Responsibility for statistical analysis, D-Writing the article, $\mathbf{E}$-Critical review, $\mathbf{F}$ - Final approval of the article 